\title{
Carrier frequency drift estimation for high mobility hierarchical-modulated MIMO-OFDM systems
}

\author{
Yang-Han Lee ${ }^{1 a)}$, Jheng-Yao Lin ${ }^{1}$, Yih-Guang Jan ${ }^{1 b)}$, \\ Wen-Ching $\mathrm{Li}^{2}$, Jing-Shown $\mathrm{Wu}^{2}$, Hen-Wai Tsao ${ }^{2}$, \\ Ming-Hsueh Chuang ${ }^{3}$, Qiang Chen ${ }^{4}$, and Qiaowei Yuan ${ }^{5}$ \\ ${ }^{1}$ Tamkang University, Tanshui District, New Taipe City, Taiwan, 25137, R.O.C. \\ ${ }^{2}$ National Taiwan University, Taipei, Taiwan, 10617, R.O.C. \\ ${ }^{3}$ National Taiwan University of Science and Technology, Taipei 106, Taiwan, R.O.C. \\ ${ }^{4}$ Tohoku University, \\ Aramaki Aza Aoba 6-6-05, Aoba-ku, Sendai-shi 980-8579, Japan \\ ${ }^{5}$ Sendai National College of Technology, \\ 4-1-16, Ayashi-shou, Aoba-ku, Sendai-shi 989-3128, Japan \\ a)yhlee@ee.tku.edu.tw \\ b)yihjan@mail.tku.edu.tw
}

\begin{abstract}
In this paper the implementation of hierarchicalmodulated pilot signals for carrier frequency drift estimation in high mobility multi-input multi-output orthogonal frequency division multiplexing (MIMO-OFDM) system is introduced. Specifically for a hierarchical-modulated 64QAM-OFDM system in which three Layers with two bits in each Layer are defined from the constellation point and the carrier frequency drift estimation is facilitated from every Layer instead of using all six constellation bits to possibly simplify the design and reduce the time in the estimation process. The carrier frequency drift estimation by using only one Layer and the system performance at the receiver terminal by utilizing this Layer's estimated carrier frequency drift are simulated and compared for various high mobile speeds in Rayleigh fading channels.
\end{abstract}

Keywords: MIMO-OFDM, Doppler Effect, hierarchical modulation, carrier frequency drift

Classification: Microwave and millimeter wave devices, circuits, and systems

\section{References}

[1] T.-D. Chiueh, OFDM Baseband Receiver Design for Wireless Communication, Wiley, 2008.

[2] P. K. Vitthaladevuni and M.-S. Alouini, "BER Computation of 4/MQAM Hierarchical Constellations," IEEE Trans. Broadcast., vol. 47, no. 3, pp. 228-239, Sept. 2001. 
[3] IEEE 802.16m System Description Document, May 2009

[Online] http://ieee802.org/16

\section{Introduction}

In a wireless cellular communication system when a mobile is moving in high speed the receiver terminal might be unable to recover the transmitted signal due to the carrier frequency drift (CFD) from Doppler Effect. In the MIMOOFDM communication system it usually uses the transmission of pilot signals as the reference signals [1] to find the channel response, to determine the possible Doppler frequency and then to compensate for the carrier frequency from this estimated carrier frequency drift. In this paper we consider in the high mobility MIMO-OFDM system in the use of hierarchical-modulated 64QAM signals as the pilot reference signals in the estimation of carrier frequency drift. In 64QAM mapping it consists of six constituent bits for every constellation point and with hierarchical modulation scheme these six constituent bits can be defined or classified into three Layers with two bits in each Layer. It then considers of using the two bits associated with one Layer instead of using all six constellation bits in the carrier frequency drift estimation to simplify the system design and reduce the system processing time.

This remaining paper is organized as in the following. In Section 2 it introduces the determination and the composition of the hierarchical-modulated 64QAM. In Section 3, the estimation of the carrier frequency drift with the utilization of only one Layer bits in a 2x2 hierarchical-modulated 64QAMOFDM system is considered and compared. And then the system performances for this $2 \times 2$ MIMO-OFDM system are simulated in a Rayleigh fading channel when a mobile is moving in several high speeds and when the estimated carrier frequency drift resulting from each Layer is offset at the receiver terminal. From these resulting simulated system performances it suggests that at certain mobile speed which particular Layer could be optimally selected in the carrier frequency drift estimation when the system suffers Doppler Effect. A conclusion is then drawn in Section 4.

\section{Hierarchical modulation}

In hierarchical modulation such as the 4/M-QAM hierarchical modulation scheme [2] it is different from the regular modulation scheme that every constituent bit of the constellation point is weighted differently in the modulation mapping. Specifically, for example, in considering 64QAM signals each constellation point is composed of six bits. From the first 2 constellation bits they determine the quadrant location of the constellation signal that is identified as 'Layer 1' quadrant and then the third and fourth bits of the constellation point determine which 'Layer 2' quadrant out of the four quadrants in the Layer 1 quadrant the constellation point is located. Finally the last 2 bits of 
each constellation point determine the location of the constellation point in the Layer 3 quadrant out of the four quadrants in the Layer 2 quadrant.

Every constellation point of $64 \mathrm{QAM}$ can then be located by three parameters $\mathrm{d}_{1}, \mathrm{~d}_{2}$ and $\mathrm{d}_{3}$ that are associated with the 'quadrants' locations as described in the above. In the in-phase axis, for example, the distances $d_{1}, d_{2}$ are the distance between the locations of Layer 1 and Layer 2 quadrants, and the distance between the locations of Layer 2 and Layer 3 quadrants respectively, while $\mathrm{d}_{3}$ is the closest distance from the constellation point along the in-phase axis to the quadrature-phase axis. The same distance parameters can be defined from the quadrature-phase axis.

In order to reduce the number of parameters used in the system design and also to simplify the distance calculation between the receiving signal and every constellation point in the estimation of system BER, we define in an alternative way by using two new parameters from $\mathrm{d}_{1}, \mathrm{~d}_{2}$ and $\mathrm{d}_{3}$ as $\lambda_{1}=\mathrm{d}_{1} / \mathrm{d}_{3}$ and $\lambda_{2}=\mathrm{d}_{2} / \mathrm{d}_{3}$ to locate every constellation point. When $\lambda_{1}$ and $\lambda_{2}$ are varied their resulting constellation locations or patterns will also be changed accordingly. There are many possible choices of $\lambda_{1}$ and $\lambda_{2}$; in this paper $\lambda_{1}$ and $\lambda_{2}$ are selected as 1.6 and 1.4 respectively and it reveals that with these selections the constellation points are non-uniformly distributed.

\section{System performance simulation}

In the system performance evaluation we simulate the system BER for a $2 \times 2$ hierarchical-modulated 64QAM-OFDM system with three mobile speeds, $250 \mathrm{~km} / \mathrm{hr}, 350 \mathrm{~km} / \mathrm{hr}$ and $500 \mathrm{~km} / \mathrm{hr}$ are considered. System parameters used in the simulation are selected from those as imposed in the $802.16 \mathrm{~m}$ specifications [3]; the subcarrier spacing is $10.935 \mathrm{KHz}$, the sampling frequency is $11.2 \mathrm{MHz}$, and the carrier frequency is $2.5 \mathrm{GHz}$. Three channel responses due to Doppler frequency drift with those three stated mobile speeds in Rayleigh fading environment are observed. The maximum Doppler frequency drifts are $578.7 \mathrm{~Hz}, 810.2 \mathrm{~Hz}$, and $1157.4 \mathrm{~Hz}$ when the mobile having speeds $250 \mathrm{~km} / \mathrm{hr}, 350 \mathrm{~km} / \mathrm{hr}$ and $500 \mathrm{~km} / \mathrm{hr}$ respectively. With respect to $10.9375 \mathrm{KHz}$ subcarrier spacing these Doppler frequency drifts have normalized Doppler frequency drifts of $0.0529,0.0741$ and 0.1058 respectively. The resulting carrier frequency drifts and the associated system BER performances by using various Layers in the estimation of carrier frequency drift in various signal-to-noise ratios (SNR) have the results as shown in Fig. 1 and the system BER performance versus SNR with the mobile speed as the simulation parameter has the simulation results as shown in Fig. 2.

From the data in the plot of Fig. 1 we build a table as in Table I that it relates the resulting system BER with the estimated normalized carrier frequency drift when different Layers are used in the carrier frequency drift estimation. The SNR is selected at $20 \mathrm{~dB}$ in setting up of this table and for other SNR values the same kind of tables can be built. In the table in the horizontal axis it has a normalized Doppler frequency drift with a spacing or resolution of 0.002 while in the vertical axis it has the normalized Doppler 


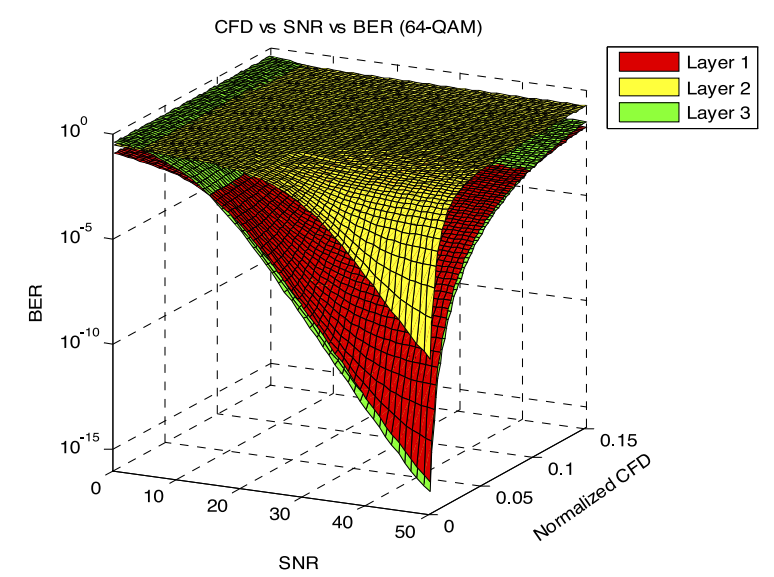

Fig. 1. Carrier Frequency Drift and BER vs. SNR in a 2x2 Hierarchical-Modulated 64QAM-OFDM System by Using Various Layer Bits in the Carrier Frequency Drift Estimation.

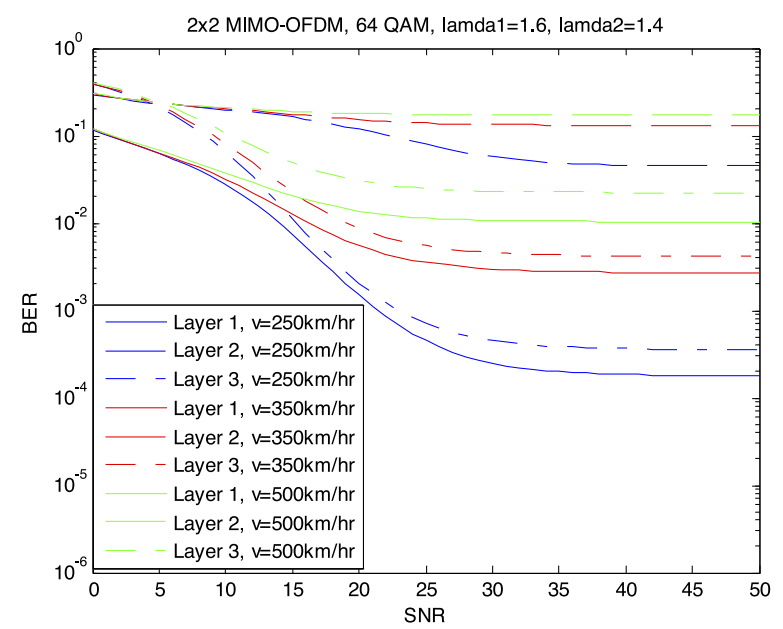

Fig. 2. A 2x2 Hierarchical-Modulated 64QAM-OFDM System Performance by Using Various Layer Bits in the Carrier Frequency Drift Estimation in Three Different Mobile Speeds.

frequency drift with a spacing or resolution of 0.02 . When a mobile travels with certain speed and using certain Layer to estimate the carrier frequency drift then it can locate a point in the table for that Layer considered and the sum of the normalized carrier frequency drifts from the horizontal and vertical axes equals to the resulting estimated carrier frequency drift; and then the associated system BER for that Layer considered can be read from the intersection of the located horizontal and vertical axes. Or on the other hand we can find the associated estimated carrier frequency drift if the system BER is known.

For example when the mobile speed is $250 \mathrm{~km} / \mathrm{hr}$, then at $\mathrm{SNR}=20 \mathrm{~dB}$ and by using Layer 1, Layer 2 and Layer 3 bits in estimating the carrier frequency drift the resulting system BERs from Fig. 2 have values of $1.50 \mathrm{E}-$ 03, 1.18E-01 and 1.90E-03 respectively. And then from Table I we can find 
Table I. Normalized Carrier Frequency Drifts when Using Different Layers in the Carrier Frequency Drift Estimation in a 2x2 Hierarchical-modulated 64 QAM-OFDM System at SNR $=20 \mathrm{~dB}$.

\begin{tabular}{|c|c|c|c|c|c|c|c|c|c|c|}
\hline \multicolumn{10}{|c|}{ Layer 1} \\
\hline CFD & 0.001 & 0.003 & 0.005 & 0.007 & 0.009 & 0.011 & 0.013 & 0.015 & 0.017 & 0.019 \\
\hline 0.02 & $4.7 \mathrm{E}-06$ & $8.9 \mathrm{E}-06$ & $1.6 \mathrm{E}-05$ & $2.7 \mathrm{E}-05$ & $4.4 \mathrm{E}-05$ & $6.8 \mathrm{E}-05$ & $1.0 \mathrm{E}-04$ & $1.5 \mathrm{E}-04$ & $2.1 \mathrm{E}-04$ & $2.8 \mathrm{E}-04$ \\
\hline 0.04 & $3.8 \mathrm{E}-04$ & $4.9 \mathrm{E}-04$ & $6.4 \mathrm{E}-04$ & $8.0 \mathrm{E}-04$ & $1.0 \mathrm{E}-03$ & $1.2 \mathrm{E}-03$ & $1.5 \mathrm{E}-03$ & $1.8 \mathrm{E}-03$ & $2.1 \mathrm{E}-03$ & $2.5 \mathrm{E}-03$ \\
\hline 0.06 & $2.8 \mathrm{E}-03$ & $3.3 \mathrm{E}-03$ & $3.7 \mathrm{E}-03$ & $4.2 \mathrm{E}-03$ & $4.7 \mathrm{E}-03$ & $5.3 \mathrm{E}-03$ & $5.9 \mathrm{E}-03$ & $6.5 \mathrm{E}-03$ & $7.1 \mathrm{E}-03$ & $7.8 \mathrm{E}-03$ \\
\hline 0.08 & $8.4 \mathrm{E}-03$ & $9.1 \mathrm{E}-03$ & $9.9 \mathrm{E}-03$ & $1.1 \mathrm{E}-02$ & $1.1 \mathrm{E}-02$ & $1.2 \mathrm{E}-02$ & $1.3 \mathrm{E}-02$ & $1.4 \mathrm{E}-02$ & $1.5 \mathrm{E}-02$ & $1.5 \mathrm{E}-02$ \\
\hline 0.10 & $1.6 \mathrm{E}-02$ & $1.7 \mathrm{E}-02$ & $1.8 \mathrm{E}-02$ & $1.9 \mathrm{E}-02$ & $2.0 \mathrm{E}-02$ & $2.1 \mathrm{E}-02$ & $2.1 \mathrm{E}-02$ & $2.2 \mathrm{E}-02$ & $2.3 \mathrm{E}-02$ & $2.4 \mathrm{E}-02$ \\
\hline \multicolumn{10}{|c|}{ Layer 2} \\
\hline CFD & 0.001 & 0.003 & 0.005 & 0.007 & 0.009 & 0.011 & 0.013 & 0.015 & 0.017 & 0.019 \\
\hline 0.02 & $3.5 \mathrm{E}-02$ & $4.3 \mathrm{E}-02$ & $5.2 \mathrm{E}-02$ & $6.0 \mathrm{E}-02$ & $6.7 \mathrm{E}-02$ & $7.5 \mathrm{E}-02$ & $8.2 \mathrm{E}-02$ & $8.9 \mathrm{E}-02$ & $9.5 \mathrm{E}-02$ & $1.0 \mathrm{E}-01$ \\
\hline 0.04 & $1.1 \mathrm{E}-01$ & $1.1 \mathrm{E}-01$ & $1.2 \mathrm{E}-01$ & $1.2 \mathrm{E}-01$ & $1.3 \mathrm{E}-01$ & $1.3 \mathrm{E}-01$ & $1.3 \mathrm{E}-01$ & $1.4 \mathrm{E}-01$ & $1.4 \mathrm{E}-01$ & $1.4 \mathrm{E}-01$ \\
\hline 0.06 & $1.5 \mathrm{E}-01$ & $1.5 \mathrm{E}-01$ & $1.5 \mathrm{E}-01$ & $1.6 \mathrm{E}-01$ & $1.6 \mathrm{E}-01$ & $1.6 \mathrm{E}-01$ & $1.6 \mathrm{E}-01$ & $1.7 \mathrm{E}-01$ & $1.7 \mathrm{E}-01$ & $1.7 \mathrm{E}-01$ \\
\hline 0.08 & $1.7 \mathrm{E}-01$ & $1.7 \mathrm{E}-01$ & $1.8 \mathrm{E}-01$ & $1.8 \mathrm{E}-01$ & $1.8 \mathrm{E}-01$ & $1.8 \mathrm{E}-01$ & $1.8 \mathrm{E}-01$ & $1.8 \mathrm{E}-01$ & $1.8 \mathrm{E}-01$ & $1.9 \mathrm{E}-01$ \\
\hline 0.10 & $1.9 \mathrm{E}-01$ & $1.9 \mathrm{E}-01$ & $1.9 \mathrm{E}-01$ & $1.9 \mathrm{E}-01$ & $1.9 \mathrm{E}-01$ & $1.9 \mathrm{E}-01$ & $1.9 \mathrm{E}-01$ & $1.9 \mathrm{E}-01$ & $2.0 \mathrm{E}-01$ & $2.0 \mathrm{E}-01$ \\
\hline \multicolumn{10}{|c|}{ Layer 3 } \\
\hline CFD & 0.001 & 0.003 & 0.005 & 0.007 & 0.009 & 0.011 & 0.013 & 0.015 & 0.017 & 0.019 \\
\hline 0.02 & $1.6 \mathrm{E}-06$ & $3.3 \mathrm{E}-06$ & $6.1 \mathrm{E}-06$ & $1.1 \mathrm{E}-05$ & $1.8 \mathrm{E}-05$ & $2.9 \mathrm{E}-05$ & $4.6 \mathrm{E}-05$ & $6.9 \mathrm{E}-05$ & $1.0 \mathrm{E}-04$ & $1.5 \mathrm{E}-04$ \\
\hline 0.04 & $2.0 \mathrm{E}-04$ & $2.8 \mathrm{E}-04$ & $3.8 \mathrm{E}-04$ & $5.0 \mathrm{E}-04$ & $6.5 \mathrm{E}-04$ & $8.3 \mathrm{E}-04$ & $1.1 \mathrm{E}-03$ & $1.3 \mathrm{E}-03$ & $1.6 \mathrm{E}-03$ & $2.0 \mathrm{E}-03$ \\
\hline 0.06 & $2.4 \mathrm{E}-03$ & $2.9 \mathrm{E}-03$ & $3.4 \mathrm{E}-03$ & $4.0 \mathrm{E}-03$ & $4.7 \mathrm{E}-03$ & $5.4 \mathrm{E}-03$ & $6.3 \mathrm{E}-03$ & $7.2 \mathrm{E}-03$ & $8.2 \mathrm{E}-03$ & $9.2 \mathrm{E}-03$ \\
\hline 0.08 & $1.0 \mathrm{E}-02$ & $1.2 \mathrm{E}-02$ & $1.3 \mathrm{E}-02$ & $1.4 \mathrm{E}-02$ & $1.6 \mathrm{E}-02$ & $1.8 \mathrm{E}-02$ & $1.9 \mathrm{E}-02$ & $2.1 \mathrm{E}-02$ & $2.3 \mathrm{E}-02$ & $2.5 \mathrm{E}-02$ \\
\hline 0.10 & $2.7 \mathrm{E}-02$ & $2.9 \mathrm{E}-02$ & $3.1 \mathrm{E}-02$ & $3.3 \mathrm{E}-02$ & $3.6 \mathrm{E}-02$ & $3.8 \mathrm{E}-02$ & $4.1 \mathrm{E}-02$ & $4.3 \mathrm{E}-03$ & $4.6 \mathrm{E}-02$ & $4.9 \mathrm{E}-02$ \\
\hline
\end{tabular}

they have the corresponding normalized Doppler frequency drift of 0.053, 0.045 and 0.057 by using Layer 1, Layer 2 and Layer 3 bits in the estimation of carrier frequency drift respectively. Similarly at $350 \mathrm{~km} / \mathrm{hr}$ mobile speed and at SNR $=20 \mathrm{~dB}$ they have normalized Doppler frequency drifts of 0.071, 0.063 and 0.079 with corresponding BERs of 5.47E-03, 1.52E-01 and 1.02E-02 respectively by using Layer 1, 2 and 3 in the estimation. For mobile speed at $500 \mathrm{~km} / \mathrm{hr}$ the normalized Doppler frequency drifts are 0.095, 0.091 and 0.105 with corresponding BERs of 1.38E-02, 1.80E-01, and 3.06E-02 respectively.

By referring to Fig. 1 and from the carrier frequency drifts as estimated for mobile at speeds at $250 \mathrm{~km} / \mathrm{hr}, 350 \mathrm{~km} / \mathrm{hr}$ and $500 \mathrm{~km} / \mathrm{hr}$, it finds that at mobile speeds of $250 \mathrm{~km} / \mathrm{hr}$ and $350 \mathrm{~km} / \mathrm{hr}$ the estimated carrier frequency drift by using Layer 1 is close to the actual carrier frequency drift than by using other two Layers and consequently at these two mobile speeds the use of Layer 1 has better estimation capability, while at $500 \mathrm{~km} / \mathrm{hr}$ the implementation of Layer 3 has better carrier frequency drift capability. From the simulation results by varying all possible mobile speeds we can devise the carrier frequency drift estimation rule for a mobile when it moves at certain speed by selecting an optimal Layer that can be used in the estimation of the carrier frequency drift without using all constellation bits.

\section{Conclusion}

In this paper we considered the estimation of the carrier frequency drift in a $2 \times 2$ hierarchical-modulated 64QAM-OFDM system when a mobile is moving. The carrier frequency drift was estimated by using only one of the three Layers of the constellation bits and each Layer generated different carrier frequency drift estimation; at certain mobile speed the use of one Layer in the carrier frequency drift estimation might get better performance than by using the other two Layers. When both the system performance 
requirement and its permitted carrier frequency drift are specified and the mobile is moving at certain speed, we can use the procedure as described in this paper to find which Layer is suitable for use in the carrier frequency drift estimation that will generate better system preformance.

\section{Acknowledgments}

This work was supported by National Science Council, R.O.C. for the financial support under Contract NSC 97-2219-E-002-027, NSC 99-2219-E-009014, NSC 97-2221-E-032-027-MY3. 